\title{
On the controllability of some quantum electro-dynamical systems *
}

\author{
Mazyar Mirrahimi \\ Ecole des Mines de Paris, Centre Automatique et Systèmes, \\ 60 Bd Saint-Michel, 75272 Paris cedex 06, FRANCE. \\ Email: mazyar.mirrahimi@ensmp.fr \\ Pierre Rouchon \\ Ecole des Mines de Paris, Centre Automatique et Systèmes, \\ 60 Bd Saint-Michel, 75272 Paris cedex 06, FRANCE. \\ Email: pierre.rouchon@ensmp.fr
}

Submitted to CDC-ECC 2005

\begin{abstract}
A result (see, e.g., [3][complement $B_{I I I}$, page 217]) of quantum electrodynamics (based on Glauber theorem) says that classical currents and sources only generate classical light (quasi-classical states of the field). This paper provides a control theoretic interpretation of this result when the classical currents and sources are considered as control inputs: the dynamics of the quantified electrodynamics field is not controllable; the controllable part is contained into the classical dynamics. This result can be seen as the infinite dimensional analogue of the following fact (see [8]): the controllable part of the quantum harmonic oscillator corresponds to the classical dynamics of the average $\langle\ddot{q}\rangle=-\langle q\rangle+u$ ( $u$ is the control input). Thus controllability can only be achieved when the field dynamics is coupled to some localized quantum dynamics. We describe here two typical models of such controlled systems: the Jaynes-Cummings model [6] (one field mode coupled to a two-level atom) and trapped ions vibrational model. Both systems correspond to experiments conducted by physicists. We recall their main approximations and validity domains. We also provide their associated formulation in terms of PDE with control.
\end{abstract}

\section{Introduction}

The interaction of photons and atoms is nowadays one of the most important topics of interest in the domain of quantum optics. Finding a reasonable and at the same time

${ }^{*}$ The authors acknowledge financial support from Action Concertée Incitative Jeunes Chercheurs, "Contrôle par laser", Ministère de la Recherche, FRANCE. simple model as well as manipulating the model are the fundamental questions for physicists. Although a lot of advances has been made in this aria (see e.g. [4] and the references herein) still remains a lot to be done especially for manipulating the systems (even very simple ones). We believe that a control point of view in this domain might be helpful for such questions (see also [7] and the references herein).

The main contribution of this paper is to study a simple model of electrodynamics in a cavity corresponding to a quantized electromagnetic field in an empty cavity where the system is controlled through a classical localized current source in the domain. This model has already been solved by physicists (see e.g. [3]) but however an understanding of the problem by the mean of control tools can be of some interest especially when more complicated models are to be taken into account. Another contribution of the paper is to present two typical systems underlying experiments conducted by physicists: control of vibrational states of trapped ions via a laser resonant with an electronic transition; control of a two-level system in an optical cavity of high quality via a resonant laser. For both systems we explain the basic modeling assumptions underlying the rotating wave approximation and provide a formulation in terms of partial differential equations. This completes [2] where, for the trapped ions system, a first order system in terms of the Lamb-Dicke parameter is discussed.

Section 2 is based on [8] where the controllability of harmonic oscillators is considered. We propose here a sightly different formulation with the use of the Heisenberg picture, unitary transformations due to Glauber and annihilation and creation operators.

Section 3 is devoted to the main goal of this paper. We adapt the formulation developed in section 2 in order to 
treat the more complicated model of an electromagnetic field in an empty cavity where the control source is assumed to be classical. We provide here a control theoretic interpretation of a classical result saying that classical currents and sources only generate classical light (quasiclassical states of the field) (see, e.g., [3][complement $B_{I I I}$, page 217])

In order to overcome the lack of controllability of the quantum degrees of freedom, one should add to the system some quantum sources. A first idea in this direction is to couple the system with a localized quantum dynamics. Jaynes-Cummings model [6] and the vibrational model for trapped ions [5] are two fundamental physical models of this kind. We introduce these models in Sections 4 and 5 and recall the main approximations and the domains of validity of these models. Finally, we give the PDE equivalent form of these systems and present the controllability problems which arise for these systems.

\section{Harmonic oscillators}

Consider a single classical harmonic oscillator (reduced frequency $\omega=1$ ):

$$
\ddot{x}=-(x-u)
$$

where $x \in \mathbb{R}$ is the state of the system and $u \in \mathbb{R}$ the one dimensional control term. One can easily see that such a system is controllable. This equation might be written as

$$
\begin{aligned}
& \dot{x}=p \\
& \dot{p}=-(x-u)
\end{aligned}
$$

where $p$ is the momentum of the system. Let us introduce the complex variable $\alpha=\frac{1}{\sqrt{2}}(x+\imath p)$ where $\imath=\sqrt{-1}$. So the equation of above will be reduced to:

$$
\imath \dot{\alpha}=\alpha-\frac{1}{\sqrt{2}} u \text {. }
$$

The quantification of such a classical using system consists in replacing the normal variables $\alpha$ and $\alpha^{*}$ (the complex conjugate of $\alpha$ ) by the well-known annihilation and creation operators $a$ and $a^{\dagger}$ with commutator equal to 1:

$$
\left[a, a^{\dagger}\right]=1 \text {. }
$$

All the physical quantities, which can be expressed as a function of $x$ and $p$ and so of $\alpha$ and $\alpha^{*}$, become operators acting in the space of the quantum states of the global system.

We can quantify system (2) with the following kinetic and potential energies:

$$
\begin{aligned}
& T=\frac{1}{2} \dot{x}^{2}=\frac{1}{2} p^{2} \\
& U=\frac{1}{2}\left(x^{2}-2 u x\right)
\end{aligned}
$$

and so the respective Hamiltonian is:

$$
H=\frac{1}{2} p^{2}+\frac{1}{2}\left(x^{2}-2 u x\right)
$$

Replacing $x$ by the position operator $X=\frac{a+a^{\dagger}}{\sqrt{2}}$ and $p$ by the momentum operator $P=\imath \frac{\partial}{\partial x}=\frac{a-a^{\dagger}}{\imath \sqrt{2}}$, we obtain the following Hamiltonian for the quantum system:

$H=-\frac{1}{2} \frac{\partial^{2}}{\partial x^{2}}+\frac{1}{2}\left(x^{2}-2 u x\right)=\left(a^{\dagger} a+\frac{1}{2}\right)-\frac{1}{\sqrt{2}} u\left(a+a^{\dagger}\right)$

The evolution of the quantum system is thus given by a bilinear controlled Schrödinger equation $(\hbar=1)$ :

$$
\imath \dot{\Psi}=\left(H_{0}+u H_{1}\right) \Psi
$$

where $H_{0}=\left(a^{\dagger} a+\frac{1}{2}\right)$ the free evolution Hamiltonian and $H_{1}=-\frac{1}{\sqrt{2}}\left(a+a^{\dagger}\right)$ the interaction operator are Hermitian operators. The state $\Psi$ can be seen as an element of $L^{2}(\mathbb{R}, \mathbb{C})$.

In [8], it is proved that (4) is not controllable: its controllable part corresponds to the average dynamics:

$$
\imath \frac{d}{d t}\langle a\rangle=\langle a\rangle-\frac{1}{\sqrt{2}} u
$$

where $\langle a\rangle=\langle\Psi, a \Psi\rangle$ denotes the average value of the physical observable $a$. We propose here bellow another formulation of the proof of this result.

Following [9], let us compute the Lie algebra generated by $\imath H_{0}$ and $\imath H_{1}$, using the standard commutation relation: $\left[a, a^{\dagger}\right]=1$. We have $\left[a^{\dagger} a, a+a^{\dagger}\right]=a^{\dagger}-a,\left[a^{\dagger} a, a^{\dagger}-a\right]=$ $a+a^{\dagger}$ and $\left[a^{\dagger}+a, a^{\dagger}-a\right]=2$. Thus this Lie algebra is of dimension 4 and the system is not controllable in a formal sense.

The purpose of this section is to show that it is possible to apply a time-dependent unitary transformation (corresponding to a controllable translation in the average system), such that in the new representation the dynamics represent a non-controllable Schrödinger equation modelling the quantum fluctuations around the average system.

Consider the unitary operator:

$$
T(t)=\exp \left[\left\langle a^{\dagger}\right\rangle a-\langle a\rangle a^{\dagger}\right]
$$

The action of such a unitary transformation on the operator $a$ is a translation by the quantity $\langle a\rangle$ (Glauber theorem):

$$
\begin{aligned}
T(t) a T^{\dagger}(t) & =a+\langle a\rangle \\
T(t) a^{\dagger} T^{\dagger}(t) & =a^{\dagger}+\left\langle a^{\dagger}\right\rangle
\end{aligned}
$$

Taking $\Phi=T(t) \Psi$ the system in the new representation 
might be written as:

$$
\begin{aligned}
& \imath \frac{d}{d t} \Phi= \\
& \left(T\left(a^{\dagger} a+\frac{1}{2}\right) T^{\dagger}-\frac{1}{\sqrt{2}} u T\left(a+a^{\dagger}\right) T^{\dagger}\right) \Phi+\left(\imath \frac{d T}{d t}\right) T^{\dagger} \Phi \\
& =\left[\left(a^{\dagger}+\left\langle a^{\dagger}\right\rangle\right)(a+\langle a\rangle)+\frac{1}{2}\right] \Phi-\frac{u}{\sqrt{2}}\left(a^{\dagger}+a+\left\langle a^{\dagger}\right\rangle+\langle a\rangle\right) \Phi \\
& +\left[\left(-\left\langle a^{\dagger}\right\rangle+\frac{u}{\sqrt{2}}\right) a-\left(\langle a\rangle-\frac{u}{\sqrt{2}}\right) a^{\dagger}\right] \Phi \\
& =\left[a^{\dagger} a+\frac{1}{2}\right] \Phi+\left[\langle a\rangle\left\langle a^{\dagger}\right\rangle-\frac{u}{\sqrt{2}}\left(\langle a\rangle+\left\langle a^{\dagger}\right\rangle\right)\right] \Phi
\end{aligned}
$$

Finally taking $\chi=S(t) \Phi$ where

$$
S(t)=\exp \imath \int^{t}\left[\langle a\rangle\left\langle a^{\dagger}\right\rangle-\frac{u}{\sqrt{2}}\left(\langle a\rangle+\left\langle a^{\dagger}\right\rangle\right)\right] d t
$$

is just a global phase change and so a unitary transformation, we obtain the following control independent Schrödinger equation:

$$
\dot{\chi}=\left[a^{\dagger} a+\frac{1}{2}\right] \chi .
$$

The dynamics of $\Psi(t, x)$ can be decomposed into two parts, a controllable one of dimension two (6), an uncontrollable one of infinite dimension (9) corresponding to the quantum fluctuations around the average dynamics.

These computations might be extended to $n$ harmonic oscillators admitting the same control $u$ but with different frequencies (see [8] for more details).

\section{Quantum electromagnetic fields with clas- sical control sources}

In this section we apply the above formulation to a more complicated physical system: a quantum electromagnetic fields in a cavity $\Omega \subset \mathbb{R}^{3}$ related by classical sources. We assume that the sources have an externally imposed motion: the currents $j$ are not affected by their radiation. In addition we suppose that the sources are macroscopic so that the quantum fluctuation of the currents around their mean value are negligible. These assumptions allow us to approximate the quantum currents $j(r)$ by well-defined functions of $r$ and $t, \mathrm{j}(r, t)\left(r \in \mathbb{R}^{3}\right.$ is the spatial variable). This section is directly inspired from the complement $B_{I I I}$ of [3].

A quantum electromagnetic field can be modelled by a set of so-called annihilation operators $a_{n}$. Each $a_{n}$ corresponds to the $n$ 'th classical mode of the electromagnetic field, $\omega_{n}$ being the associated frequency. One postulates the following commutation relations:

$$
\begin{aligned}
& {\left[a_{n}, a_{k}\right]=\left[a_{n}^{\dagger}, a_{k}^{\dagger}\right]=0} \\
& {\left[a_{n}, a_{k}^{\dagger}\right]=\delta_{n k}}
\end{aligned}
$$

The $\delta_{n k}$ indicates that the operators of two different modes commute.

The evolution of the fields in the Heisenberg picture is then given by:

$$
\dot{a}_{n}+\imath \omega_{n} a_{n}=\mathrm{j}_{n}
$$

where

$$
\mathrm{j}_{n}=\frac{\imath}{\sqrt{2}}\left\langle\frac{\partial \mathrm{j}}{\partial t}, \phi_{n}\right\rangle_{L^{2}(\Omega)}
$$

$\phi_{n}(r)$ being the mode $n$ of the operator $-\triangle$ in the compact smooth domain $\Omega$ with Dirichlet's boundary conditions. The Hamiltonian of the system is then given by:

$$
H=\sum_{n} \omega_{n}\left(a_{n}^{\dagger} a_{n}+\frac{1}{2}\right)-\mathrm{j}_{n}\left(a_{n}+a_{n}^{\dagger}\right)
$$

Formally, the state $\Psi$ belongs to a state-space made of an infinite tensor product of $L^{2}(\mathbb{R}, \mathbb{C}), \bigotimes_{n} L^{2}(\mathbb{R}, \mathbb{C})$. The system's evolution is thus given by the following bilinear controlled schrödinger equation:

$$
\imath \dot{\Psi}=H \Psi .
$$

Using classical Ehrenfest theroem (or equations (10)), we have the following dynamics for the mean value of the fields:

$$
\begin{aligned}
\frac{d}{d t}\left\langle a_{n}+a_{n}^{\dagger}\right\rangle & =\frac{\omega_{n}}{\imath}\left\langle a_{n}-a_{n}^{\dagger}\right\rangle \\
\frac{d}{d t}\left\langle a_{n}-a_{n}^{\dagger}\right\rangle & =\frac{\omega_{n}}{\imath}\left\langle a_{n}+a_{n}^{\dagger}\right\rangle-2 \frac{\mathrm{j}_{n}}{\imath}
\end{aligned}
$$

These dynamics correspond to the classical evolution of the mean value of the electric and magnetic fields. Since $-\omega_{n}^{2}$ being the $n$ 'th eigenvalue of the operator $-\triangle$ in $\Omega$ with Dirichlet's boundary conditions, the equations (12) is the projection on mode $n$ of the following wave equation:

$$
\ddot{E}=\triangle E-\sqrt{2} \frac{\partial}{\partial t} \mathrm{j} \quad \text { in } \quad(0, T) \times \Omega
$$

with null boundary conditions. In fact the standard model for a classical electromagnetic field in a bounded domain with perfect conductor boundaries is given by the following Maxwell equations (a simple computation shows that these equations lead to (13)):

$$
\begin{aligned}
\nabla \cdot E(r, t) & =0 \\
\nabla \cdot B(r, t) & =0 \\
\nabla \times E(r, t) & =-\frac{\partial}{\partial t} B(r, t) \\
\nabla \times B(r, t) & =\frac{\partial}{\partial t} E(r, t)+\sqrt{2} \mathrm{j}
\end{aligned}
$$

Controllability of the classical system (13) where the control term appears in a localized current source $\mathrm{j}$, has already been studied (see e.g. [1, 10] and the references herein). 
We will show that the quantized dynamics of (13) (the control j remains classical) is not controllable: one cannot control the quantum fluctuation of the electrodynamic field with a classical control.

Consider the following unitary transformation on $\bigotimes_{n} L^{2}(\mathbb{R}, \mathbb{C})$ :

$$
T=\bigotimes_{n} T_{n}
$$

where

$$
T_{n}=\exp \left[\left\langle a_{n}^{\dagger}\right\rangle a_{n}-\left\langle a_{n}\right\rangle a_{n}^{\dagger}\right] .
$$

We have (Glauber theorem):

$$
\begin{aligned}
& T_{n} a_{n} T_{n}^{\dagger}=a_{n}+\left\langle a_{n}\right\rangle \\
& T_{k} a_{n} T_{k}^{\dagger}=a_{n} \quad \text { for } \quad k \neq n
\end{aligned}
$$

Taking $\Phi=T(t) \Psi$, just as in the Section 2, we have:

$$
\begin{aligned}
\imath \dot{\Phi}= & {\left[\sum_{n} \omega_{n}\left(a_{n}^{\dagger} a_{n}+\frac{1}{2}\right)\right] \Phi } \\
& +\left[\sum_{n} \omega_{n}\left\langle a_{n}\right\rangle\left\langle a_{n}^{\dagger}\right\rangle-\mathrm{j}_{n}\left(\left\langle a_{n}\right\rangle+\left\langle a_{n}^{\dagger}\right\rangle\right)\right] \Phi
\end{aligned}
$$

and then taking $\chi=S(t) \Phi$ where

$S(t)=\exp \left(\imath \int^{t}\left[\sum_{n} \omega_{n}\left\langle a_{n}\right\rangle\left\langle a_{n}^{\dagger}\right\rangle-\mathrm{j}_{n}\left(\left\langle a_{n}\right\rangle+\left\langle a_{n}^{\dagger}\right\rangle\right)\right] d t\right)$

is just a global phase change and so a unitary transformation, we obtain the following control independent Schrödinger equation:

$$
\iota \dot{\chi}=\left[\sum_{n} \omega_{n}\left(a_{n}^{\dagger} a_{n}+\frac{1}{2}\right)\right] \chi .
$$

The observable corresponding to the electro-magnetic field is thus the sum of two contributions: its average value described by (13) those controllability is relevant of the sharp sufficient conditions given in [1], and the vacuum fluctuations described by (16) and that are not controllable because their dynamics are completely independent of the control.

\section{Jaynes-Cummings model with control}

As we have seen, we cannot control the quantum fluctuations of an electrodynamic field in a cavity via classical currents. It is thus necessary to add inside the cavity a localized quantum dynamics coupled to the field. The JaynesCummings model is one of the simplest models describing the coupling of a two-level system with an electrodynamic cavity: the Bohr frequency $\omega_{b}$ is close to an isolated cavity mode of frequency $\omega_{c}\left(\left|\omega_{b}-\omega_{c}\right| \ll \omega_{b}\right)$. The state-space of the system is made of the tensor product of the infinite dimensional state-space of a harmonic oscillator, $L^{2}(\mathbb{R}, \mathbb{C})$, and of the two-dimensional state-space of the atom, $\mathbb{C}^{2}$. Such a state-space is equivalent to $L^{2}(\mathbb{R}, \mathbb{C}) \times L^{2}(\mathbb{R}, \mathbb{C})$. Let us note by $\Psi=\left(\Psi_{g}, \Psi_{e}\right) \in L^{2}(\mathbb{R}, \mathbb{C}) \times L^{2}(\mathbb{R}, \mathbb{C})$ the state of the system: $\Psi=\Psi_{g}|g\rangle+\Psi_{e}|e\rangle$ where $|g\rangle$ and $|e\rangle$ are respectively the ground state and the excited state of the system. If we still assume that we have at our disposal a classical control $u$ (classical current or coherent laser light) and when we consider only the isolated resonant cavity mode $\left(\omega_{c} \approx \omega_{b}\right)$, then the cavity Hamiltonian may be written as

$$
H_{c}=\omega_{c} a^{\dagger} a-u\left(a+a^{\dagger}\right)
$$

The internal Hamiltonian of the atom is given by:

$$
H_{a}=\frac{\omega_{b}}{2}(|e\rangle\langle e|-| g\rangle\langle g|)=\frac{\omega_{b}}{2} \sigma_{z}
$$

where $\sigma_{x}, \sigma_{y}$ and $\sigma_{z}$ are the so-called Pauli matrices. Finally the interaction Hamiltonian between the atom and the cavity is given by:

$$
H_{\text {int }}=\frac{\Omega}{2}\left(a+a^{\dagger}\right)(|e\rangle\langle g|+| g\rangle\langle e|)=\frac{\Omega}{2}\left(a+a^{\dagger}\right) \sigma_{x}
$$

where $\Omega$ is the vacuum Rabi frequency $\left(\Omega \ll \omega_{c}, \omega_{b}\right)$. The system Hamiltonian is the sum of all these Hamiltonians: $H_{c}+H_{a}+H_{i n t}$. So a first simplification consists in considering the system in the rotating wave approximation (interaction frame). We set

$$
\Psi=\exp \left(-\imath \omega_{b} t a^{\dagger} a\right) \exp \left(-l \frac{\omega_{b}}{2} t \sigma_{z}\right) \Phi .
$$

A simple computation shows that

$$
\begin{aligned}
& \exp \left(\imath \omega_{b} t a^{\dagger} a\right) a \exp \left(-\imath \omega_{b} t a^{\dagger} a\right)=e^{-\imath \omega_{b} t} a \\
& \exp \left(\imath \frac{\omega_{b}}{2} t \sigma_{z}\right) \sigma_{x} \exp \left(-\imath \frac{\omega_{b}}{2} t \sigma_{z}\right)=e^{-\imath \omega_{b} t}|g\rangle\left\langle e\left|+e^{\imath \omega_{b} t}\right| e\right\rangle\langle g|
\end{aligned}
$$

where we have used the fact that the operators $a^{\dagger} a$ and $a a^{\dagger}$ commute. These relation yields to a new formulation for the system's Hamiltonian given by

$$
\begin{aligned}
H= & \left(\omega_{c}-\omega_{b}\right) a^{\dagger} a-u\left(e^{-\imath \omega_{b} t} a+e^{\imath \omega_{b} t} a^{\dagger}\right) \\
& +\frac{\Omega}{2}\left(e^{-\imath \omega_{b} t} a+e^{\imath \omega_{b} t} a^{\dagger}\right)\left(e^{-\imath \omega_{b} t}|g\rangle\left\langle e\left|+e^{\imath \omega_{b} t}\right| e\right\rangle\langle g|\right) .
\end{aligned}
$$

Finally, setting $u=v e^{-\imath \omega_{b} t}+v^{*} e^{\imath \omega_{b} t}$ with $v$ a slowly varying complex amplitude (new control $v \in \mathbb{C}$ ), we get neglecting oscillating terms $e^{ \pm 2 \imath \omega_{b} t}$, the controlled Jaynes-Cummings Hamiltonian (in the interaction representation)

$H_{J C}=\left(\omega_{c}-\omega_{b}\right) a^{\dagger} a+\frac{\Omega}{2}\left(a|e\rangle\left\langle g\left|+a^{\dagger}\right| g\right\rangle\langle e|\right)-\left(v a^{\dagger}+v^{*} a\right)$ 
where $\omega_{c}-\omega_{b}$ has the same magnitude as $\Omega$. Assume now that $\omega_{c}=\omega_{b}$. We get in PDE language and up to a scaling, the bellowing system:

$$
\begin{aligned}
& l \frac{\partial \psi_{g}}{\partial t}=\left(v_{1} x+\imath v_{2} \frac{\partial}{\partial x}\right) \psi_{g}+\left(x+\frac{\partial}{\partial x}\right) \psi_{e} \\
& \iota \frac{\partial \psi_{e}}{\partial t}=\left(x-\frac{\partial}{\partial x}\right) \psi_{g}+\left(v_{1} x+\imath v_{2} \frac{\partial}{\partial x}\right) \psi_{e}
\end{aligned}
$$

where $v=v_{1}+\imath v_{2} \in \mathbb{C}$ is the control. A unitary transformation similar to the one used in Section 2 yields to another version of this control problem. In this aim, we consider $w \in \mathbb{C}$ so that it verifies $l \frac{d}{d t} w=-v$ and we take the unitary transformation $T(t)=\exp \left[w^{*} a-w a^{\dagger}\right]$. Transforming the system by this unitary transformation and up to a global phase change, we obtain the new version:

$$
\tilde{H}_{J C}=\frac{\Omega}{2}\left[(a+w)|e\rangle\left\langle g\left|+\left(a^{\dagger}+w^{*}\right)\right| g\right\rangle\langle e|\right]
$$

where $w \in \mathbb{C}$ is the new control corresponding to the integral of the physical control $v$.

In PDE configuration and up to an appropriate scaling, the system's evolution may be written as follows:

$$
\begin{aligned}
& l \frac{\partial \Psi_{g}}{\partial t}=\left(x+w_{1}+\frac{\partial}{\partial x}+\imath w_{2}\right) \Psi_{e} \\
& \iota \frac{\partial \Psi_{e}}{\partial t}=\left(x+w_{1}-\frac{\partial}{\partial x}-\imath w_{2}\right) \Psi_{g}
\end{aligned}
$$

where $w=w_{1}+\imath w_{2} \in \mathbb{C}$ is the control (time integral of the physical control $v$ the amplitude and phase modulation).

The Jaynes-Cummings system is equivalent to

$$
\iota \frac{d}{d t} \Psi=\left(H_{0}+w_{1} H_{1}+w_{2} H_{2}\right) \Psi
$$

with

$$
H_{0}=X \sigma_{x}-P \sigma_{y}, \quad H_{1}=\sigma_{x}, \quad H_{2}=\sigma_{y}
$$

and

$$
X=\frac{a+a^{\dagger}}{\sqrt{2}}, \quad P=\frac{a-a^{\dagger}}{l \sqrt{2}} .
$$

The Lie algebra spanned by $\imath H_{0}, \imath H_{1}$ and $\iota H_{2}$ is infinite dimensional now. But one can prove that the linear tangent approximation around any eigen-state of $H_{0}+w_{1} H_{1}+$ $w_{2} H_{2}$ for any control value $w_{1}$ and $w_{2}$ is not controllable. The controllability of such a system seems to be an interesting problem both from the mathematical and practical points of view.

\section{Laser control of trapped ions}

We refer to [5] for a very nice presentation of such system. Its Hamiltonian is quite similar to the one presented for the Jaynes-Cummings model:

$$
\begin{aligned}
H= & \Omega\left(a^{\dagger} a+1 / 2\right)+\frac{\omega_{b}}{2}(|e\rangle\langle e|-| g\rangle\langle g|) \\
& +\left[u e^{l(\omega t-k X)}+u^{*} e^{-l(\omega t-k X)}\right](|e\rangle\langle g|+| g\rangle\langle e|)
\end{aligned}
$$

with $k X=\eta\left(a+a^{\dagger}\right)$ and where $\eta \ll 1$ is the Lamb-Dicke parameter. Here the control $u \in \mathbb{C}$ consists in the amplitude and phase modulations of the laser of frequency $\omega$ that is quasi-resonant via the internal dynamics $\omega_{b}: \omega \approx \omega_{b}$. The vibration frequency $\Omega$ is much smaller than $\omega$ and corresponds to a harmonic potential within which the ions are trapped: $\Omega \ll \omega \approx \omega_{b}$.

Assuming $\omega=\omega_{b}$ and setting $\Psi=\exp \left(-\imath \omega t \sigma_{z} / 2\right) \Phi$, the Hamiltonian becomes:

$H=\Omega\left(a^{\dagger} a+1 / 2\right)+$
$\left[u e^{l\left(\omega t-\eta\left(a+a^{\dagger}\right)\right)}+u^{*} e^{-l\left(\omega t-\eta\left(a+a^{\dagger}\right)\right)}\right]\left(e^{-l \omega t}|g\rangle\left\langle e\left|+e^{\imath \omega t}\right| e\right\rangle\langle g|\right)$

Finally using the rotating wave approximation and neglecting highly oscillating terms of the form $e^{ \pm 2 \imath \omega t}$, we obtain the following averaged Hamiltonian:

$\tilde{H}=\Omega\left(a^{\dagger} a+1 / 2\right)+u e^{-\imath \eta\left(a+a^{\dagger}\right)}|g\rangle\left\langle e\left|+u^{*} e^{\imath \eta\left(a+a^{\dagger}\right)}\right| e\right\rangle\langle g|$.

This Hamiltonian in a PDE configuration corresponds to the following evolution equation $(\eta \mapsto \eta \sqrt{2})$ :

$$
\begin{aligned}
l \frac{\partial \psi_{g}}{\partial t} & =\frac{\Omega}{2}\left(x^{2}-\frac{\partial^{2}}{\partial x^{2}}\right) \psi_{g}+u e^{-\imath \eta x} \psi_{e} \\
l \frac{\partial \psi_{e}}{\partial t} & =u^{*} e^{\imath \eta x} \psi_{g}+\frac{\Omega}{2}\left(x^{2}-\frac{\partial^{2}}{\partial x^{2}}\right) \psi_{e}
\end{aligned}
$$

where $u \in \mathbb{C}$ is the control and $\eta \ll 1$. Controllability of this system is another interesting problem to be treated, as it has already been mentioned in [2], where $e^{\imath \eta x}$ is approximated by $1+\imath \eta x$.

\section{Discussions and Conclusion}

As we have seen, we cannot control the quantum fluctuations of an electrodynamic field in a cavity via classical currents. It is thus necessary to add inside the cavity a localized quantum dynamics coupled to the field. The JaynesCummings and trapped ions model are some typical models where quantum dynamics are coupled to a quantized electrodynamic field. Such systems show more interesting controllability properties than a quantized electrodynamic field alone. The exact controllability or even the approximate controllability of such systems are questions that are motivated by physical interest and also that seem to be challenging from the mathematical point of view. 


\section{References}

[1] C. Bardos, G. Lebeau, and J. Rauch. Sharp sufficient conditions for the observation, control and stabilization of waves from the boundary. SIAM J. Control Opt., 30:1024-1065, 1992.

[2] R.W. Brockett, C. Rangan, and A.M. Bloch. The controllability of infinite quantum systems. In Control and Decision Conference, Hawai, pages 428-433, 2003.

[3] C. Cohen-Tannoudji, J. Dupont-Roc, and G. Grynberg. Photons and Atoms: Introduction to Quantum Electrodynamics. Wiley, 1989.

[4] C. Cohen-Tannoudji, J. Dupont-Roc, and G. Grynberg. Atom-Photon interaction: Basic Processes and Applications. Wiley, 1992.

[5] S. Haroche. Contrôle de la décohérence: théorie et expériences, 2004. Notes de cours, Collège de France. http://www.lkb.ens.fr/recherche/qedcav/college/college.html.

[6] E.T. Jaynes and F.W. Cummings. Comparison of quantum and semiclassical radiation theories with application to the beam maser. Proceedings of the IEEE, 51(1):89-109, 1963.

[7] C. Le Bris, Y. Maday, and G. Turinici. Towards efficient numerical approaches for quantum control. preprint, October 2004.

[8] M. Mirrahimi and P. Rouchon. Controllability of quantum harmonic oscillators. IEEE Trans Automatic Control, 49(5):745-747, 2004.

[9] V. Ramakrishna, M. Salapaka, M. Dahleh, and H. Rabitz. Controllability of molecular systems. Phys. Rev. A, 51(2):960-966, 1995.

[10] X. Zhang and E. Zuazua. Controllability of nonlinear partial differential equations. In Proceedings of the Second IFAC Workshop on Lagrangian and Hamiltonian methods in Nonlinear Control, Sevilla, pages 269-274, 2003. 